\title{
A tiered-layered-staged model for informed consent in personal genome testing
}

\author{
Eline M Bunnik ${ }^{\star}, 1$ A Cecile JW Janssens ${ }^{2}$ and Maartje HN Schermer ${ }^{1}$
}

In recent years, developments in genomics technologies have led to the rise of commercial personal genome testing (PGT): broad genome-wide testing for multiple diseases simultaneously. While some commercial providers require physicians to order a personal genome test, others can be accessed directly. All providers advertise directly to consumers and offer genetic risk information about dozens of diseases in one single purchase. The quantity and the complexity of risk information pose challenges to adequate pre-test and post-test information provision and informed consent. There are currently no guidelines for what should constitute informed consent in PGT or how adequate informed consent can be achieved. In this paper, we propose a tiered-layered-staged model for informed consent. First, the proposed model is tiered as it offers choices between categories of diseases that are associated with distinct ethical, personal or societal issues. Second, the model distinguishes layers of information with a first layer offering minimal, indispensable information that is material to all consumers, and additional layers offering more detailed information made available upon request. Finally, the model stages informed consent as a process by feeding information to consumers in each subsequent stage of the process of undergoing a test, and by accommodating renewed consent for test result updates, resulting from the ongoing development of the science underlying PGT. A tieredlayered-staged model for informed consent with a focus on the consumer perspective can help overcome the ethical problems of information provision and informed consent in direct-to-consumer PGT.

European Journal of Human Genetics (2013) 21, 596-601; doi:10.1038/ejhg.2012.237; published online 21 November 2012

Keywords: personal genome testing; informed consent; ethical issues; complex diseases

\section{INTRODUCTION}

For a few years, a new generation of so-called personal genome testing (PGT) companies has been marketing genome-wide SNP analysis and whole genome sequencing directly to consumers. These companies offer personal risks for dozens of diseases simultaneously, including cardiovascular diseases, type 2 diabetes, psychiatric conditions and many types of cancer. (Pathway: https://www.pathway.com; Knome: http://www.knome.com; Counsyl: https://www.counsyl.com; 23andme: http://www.23andme.com; deCODEme: http://www.decodeme.com) Consumers can obtain this information through web-based services with (Pathway: https://www.pathway.com; Counsyl: https://www. counsyl.com) or without (23andme: http://www.23andme.com; deCODEme: http://www.decodeme.com) the involvement of a medical professional in the signing off on the test order. Consumers take a cheek swab sample at home, send their sample to a molecular genetic testing laboratory through the mail and receive their genetic test results on a secure personal webpage.

Informed consent is an essential ethical requirement in genetic testing $^{1}$ and entails more than the signing of a consent form or the ticking of a checkbox. ${ }^{2}$ From an ethical perspective, informed consent is a communicative process of providing intelligible, preferably tailored information, checking whether the patient—or in the case of PGT, the consumer-has understood all relevant information, complementing information found to be lacking, seeing again that all informational needs have been met, and finally, asking for informed consent. Most ethical conceptions of informed consent aim at patients' self-determination, autonomous decision-making and right to choose, ${ }^{3}$ and at the protection against harm. ${ }^{4}$ As such, they are much more demanding than legal 'narration-followed-by-signature' conceptions of informed consent. ${ }^{5}$ Most ethical theories of informed consent agree that informed consent has at least three preconditions: information, comprehension and voluntariness. ${ }^{6}$ At minimum, persons giving informed consent should be legally and cognitively capable of consenting, they should be free from external control, and they should have adequate information and understanding of what it is they are consenting to. Voluntariness as an ethical requirement may be violated when consumers send in samples from third parties without their consent. As an online health service, PGT can be vulnerable to this form of misuse, which should naturally be discouraged. For the remainder of this paper we will however not focus on voluntariness, but on information and comprehension, which are becoming increasingly pressing issues for PGT.

A traditional ethical conception of informed consent in the context of clinical genetic testing requires consumers to receive pre-test information about the test, the disease tested for, possible outcomes and their significance, implications, limitations and risks of testing, and many other relevant aspects. ${ }^{7}$ This conception cannot be translated directly to the very different context of PGT: it will be

${ }^{1}$ Department of Medical Ethics and Philosophy of Medicine, Erasmus University Medical Center, Rotterdam, The Netherlands; ${ }^{2}$ Department of Epidemiology, Erasmus University Medical Center, Rotterdam, The Netherlands

${ }^{*}$ Correspondence: EM Bunnik, Department of Medical Ethics and Philosophy of Medicine, Erasmus University Medical Center Rotterdam, Office AE-339, PO Box 2040, Rotterdam, 3000 CA, The Netherlands. Tel: +31 10 7043062; Fax: +31 10 7044735; E-mail e.bunnik@erasmusmc.nl

Received 19 June 2012; revised 3 September 2012; accepted 27 September 2012; published online 21 November 2012 
difficult, if not impossible, for providers to produce and for consumers-given the limited genetic health literacy among many of them ${ }^{8}$ - to process detailed, specific information on large numbers of tests and diseases at the same time. Empirical research has not yet brought consensus on whether consumers understand important information about the (limited) clinical utility of a PGT.9,10 Especially in a direct-to-consumer context and without the help of a medical professional, geneticist or genetic counsellor, information about PGT is likely to be misunderstood by consumers. ${ }^{11,12}$ Therefore, the provision of adequate information and informed consent are among the main concerns in PGT. ${ }^{13,14}$

Current guidelines for information provision in $\mathrm{PGT}^{14,15}$ do not distinguish between information provision and informed consent. They list a lot of information to be mentioned on providers' websites, but not all of this information pertains to informed consent. By overloading consumers with less relevant information, informational practices modelled on these guidelines may fail to convey the important information elements that are necessary for informed consent. None of the guidelines specifies how adequate informed consent can be obtained.

The quality of information provision in PGT is high for some of the leading companies, but not perfect. ${ }^{16}$ Most companies provide abundant information, including educational materials and references to the scientific literature. (Pathway: https://www.pathway.com; Knome: http://www.knome.com; Counsyl: https://www.counsyl.com; 23andme: http://www.23andme.com) Yet, abundant information does not equal adequate or relevant information and may mislead or overwhelm consumers while failing to convey the key messages about PGT. Company websites headline 'improve your health', 'plan for the future', 'take a more active role in managing your health', or '23andme saved my life,' (23andme: http://www.23andme.com) thereby overstating the clinical utility of a large proportion of their testing offer. They highlight the benefits of a PGT, while information about risks and limitations is often difficult to locate. ${ }^{16,17}$ Information about risks is mentioned in 'Terms of Service' or 'Terms of Use' agreements (Pathway: https://www.pathway.com; Counsyl: https://www.counsyl. com; 23andme: http://www.23andme.com) in which legal requirements are laid out and which companies use instead of informed consent procedures. Terms of Service agreements however are notoriously lengthy and are unlikely to be read completely by each customer. ${ }^{15}$ Furthermore, these agreements can be said to aim at the legal protection of companies rather than of consumers. They are not necessarily designed to stimulate consumer understanding, nor do they automatically constitute informed consent.

There is room for improvement in information provision ${ }^{16,17}$ and informed consent in a large proportion of PGTs offered. This paper proposes a model for informed consent that is suitable to handling the quantity and complexity of information in PGT. The model is meant for online use and can be applied to both direct-to-consumer and physician-mediated forms of commercial PGT based on either genome-wide scans or exome or whole genome sequencing. The advent of exome or whole genome sequencing technologies in PGT may further expand the testing offer and increase the clinical validity and utility of some of the findings, and may thus exacerbate the need for a new model for informed consent. We start from the assumption that it may not be necessary or desirable to legally require professional counselling or to ban direct access to PGT, and that although there is a moral obligation for providers to facilitate informed consent, the responsibility of actually making informed, autonomous decisions regarding PGT, rests with the consumer. ${ }^{18}$

\section{MODELS FOR INFORMED CONSENT}

A wide range of models for and approaches to informed consent have been developed in the history of medicine. ${ }^{4}$ Two main contrasting approaches are specific consent, which in the context of genetic testing requires consumers to be provided with elaborate and detailed information about the testing procedure, the diseases tested for and implications of testing, and generic consent, which focuses on general information and common-denominator issues, such as general characteristics of genetics and genetic testing, and common features of the diseases tested for. ${ }^{19,20}$ Neither approach will be completely suitable to PGT, because they both fail to promote two important ethical aims of informed consent: the enabling of autonomous choice and the protection against harms. ${ }^{21}$ Because of the complexity and the quantity of the information offered in PGT, informed consent cannot be fully specific. It will be impossible to provide consumers with detailed information on all relevant aspects on all diseases tested for in a PGT. Overloading consumers with detailed information will undermine autonomous choice. On the other hand, informed consent should not be too generic either. Consumers should at least know what test results they will receive and be given the opportunity to opt out of receiving test results which they anticipate may harm them or which they do not wish to receive. ${ }^{18}$

\section{A TIERED, LAYERED AND STAGED MODEL FOR INFORMED CONSENT}

An ethical approach to informed consent underlines the importance of ensuring that consumers understand the clinical and emotional impact PGT may have on them, and the implications for their personal lives as well as for the lives of their family members. Informed consent in PGT should aim at the provision of both complete information (specific consent) and understandable information (generic consent). The proposed model consists of a combination of tiered, layered and staged models for informed consent, three existing approaches to informed consent ${ }^{7,22,23}$ that all attempt to provide information which is as complete as possible while remaining understandable. Each of the three models will be described and applied to PGT.

\section{Tiered consent}

Tiered consent is differentiated consent. The broad PGT offer can be subdivided into tiers or categories of traits and diseases. Informed consent can then be given for specified categories of diseases rather than for the complete package. Differentiation of the testing offer can help consumers make deliberate choices with regard to the information they do or do not want to receive.

Tiered consent is currently widely used for biobanks and genomic databases $^{22,24}$ and for neonatal screening programmes. ${ }^{25} \mathrm{~A}$ few 'binning' or 'packaging' models have recently been suggested for the return of results to research participants and for the interpretation of whole genome sequencing. ${ }^{26-28}$ A 'packages' model distinguishes a default package of research results and optional packages. ${ }^{26}$ The 'default package' contains information that should always be reported back to participants such as directly life-saving information and other information of high clinical utility indicating serious health problems. ${ }^{26}$ The optional packages include data of moderate clinical validity, data of reproductive significance and data of 'personal or recreational' interest. One version of the 'binning' model consists of five bins, in which the first bin contains medically actionable results, the second results that have implications for family planning, the third information that may be sensitive and unwanted (such as APOE results), the fourth information that has clinical validity for diseases 
for which there are no therapeutic or preventive options and the fifth bin, finally, contains all other 'results' or all data for which a clinical interpretation is lacking or uncertain. ${ }^{28}$ In these models, the purpose of the test is used as a criterion, for example: carrier status results that can be used for reproductive decision-making are assigned a category of their own. Interestingly, in the latter model the bins are defined according to the emotional impact of results: there are separate bins for sensitive or unwanted results and results lacking actionability. For consumers, the purpose and the emotional impact of a test are likely to be important criteria in deciding whether or not to take the test-and to give informed consent. Empirical research is needed to examine what criteria consumers find important or meaningful to their decision-making processes.

In PGT, a tiered model for informed consent can be used to distinguish between categories of diseases on the basis of both test characteristics (eg clinical validity and utility) and disease characteristics (eg severity, actionability, age of onset and the somatic or psychiatric nature of the disease), ${ }^{29}$ as these characteristics are associated with different clinical, psychological and social implications for consumers. In a tiered process, consumers could for instance choose to obtain only testing of high clinical validity and utility for its medical value or-if they wish-only testing for non-medical traits for its informational or 'curiosity' value. Alternatively, they could choose to purchase testing for somatic diseases only and leave out psychiatric diseases, or for early-onset diseases only, not for late-onset diseases. Combinations of test characteristics and disease characteristics may also be appropriate, for example: consumers could indicate that for very severe diseases, they would only want to know their genetic risk if the test is of high clinical validity. Providers of PGT could thus structure their testing offer beforehand into tiers that are meaningful for consumers, such as purposes of testing, severity of the diseases tested for, actionability and emotional impact of test results.

At first glance, tiered consent might seem to pose practical challenges to the business models of PGT providers. It might require them to tailor their services according to consumers' preferences. Some companies have already differentiated their services and offer 'cardio scans' alongside 'complete scans' (deCODEme: http://www.decodeme.com) or pre-pregnancy planning services alongside drug response services. (Pathway: https://www.pathway.com). Alternatively, providers could conduct complete scans for all customers while offering the possibility to opt out of receiving results for certain tiers.

\section{Layered consent}

Layered consent distinguishes between different layers of information. The first layer pertains directly to informed consent and is indispensable to informed consent. This information is explicitly offered to all consumers and is kept minimal in order to increase the effectiveness of its communication. There may be other information elements that should be offered as part of a broader, general provision of information, ${ }^{14,15}$ but these should be made available in second and further layers of the information provision process. The concept of layered consent is based upon the idea of extendable information: ${ }^{4}$ some information is offered to all consumers, whereas other, more detailed information is accessible for consumers who actively seek it. Information provision in public screening activities is often layered. ${ }^{23}$

An ethical approach to informed consent in PGT acknowledges that the first layer should contain limited information in order to remain comprehensible and manageable, and to avoid information overload. ${ }^{30}$ Many consumers will only read what is presented to them first and will not seek additional information. ${ }^{31}$ Therefore, the first layer should contain all crucial information, all the key messages about
PGT that are necessary for consumers' understanding of PGT and for their decision whether or not to take the test. For this reason, existing guidelines for information provision in PGT may be too encompassing as they include specific information such as the location of the provider, funding arrangements and the evidence on which interpretations of the test results are based. ${ }^{14,15}$ Although this information may need to be available on PGT providers' websites, it is not part of the informed consent process itself and should not be presented in the first layer, for it will distract consumers from what they need to know in order to consent to PGT.

Informational needs for informed consent in PGT have not yet been established. Following existing guidelines for information provision in $\mathrm{PGT}^{14,15}$ and in clinical genetic testing, ${ }^{7}$ we expect that consumers need to know at least the following eight information elements in order to give informed consent: the purpose of the test, the target group, limitations of the tests, risks and implications of testing, tiers of the PGT, potential follow-up, data protection, and where to find further and independent information (see Table 1). By way of illustration, we discuss the most important elements briefly.

Consumers should understand the purpose of a genetic test at the outset. ${ }^{32}$ PGT services are marketed for a variety of purposes, ranging from prediction of risks for complex diseases (deCODEme: http://www.decodeme.com) and pre-conception carrier screening (Counsyl: https://www.counsyl.com) to pharmacogenomic information, information on other, non-medical phenotypic traits, such as ear wax type or eye colour, (23andme: http://www.23andme.com) and nutrigenomic information (Pathway: https://www.pathway.com). In order to manage consumers' expectations and help them understand correctly the nature of the PGT on offer, the purpose(s) of PGT should be clearly addressed. In tandem with the purpose, the target group(s) should be made explicit in the first layer. ${ }^{32}$ Possible target groups are couples who intend to conceive, (Counsyl: https:// www.counsyl.com) healthy, asymptomatic adult individuals ${ }^{33}$ who wish to know their genetic susceptibilities to complex diseases, (23andme: http://www.23andme.com; deCODEme: http://www.

Table 1 A proposed contents of the first layer of the information provision process

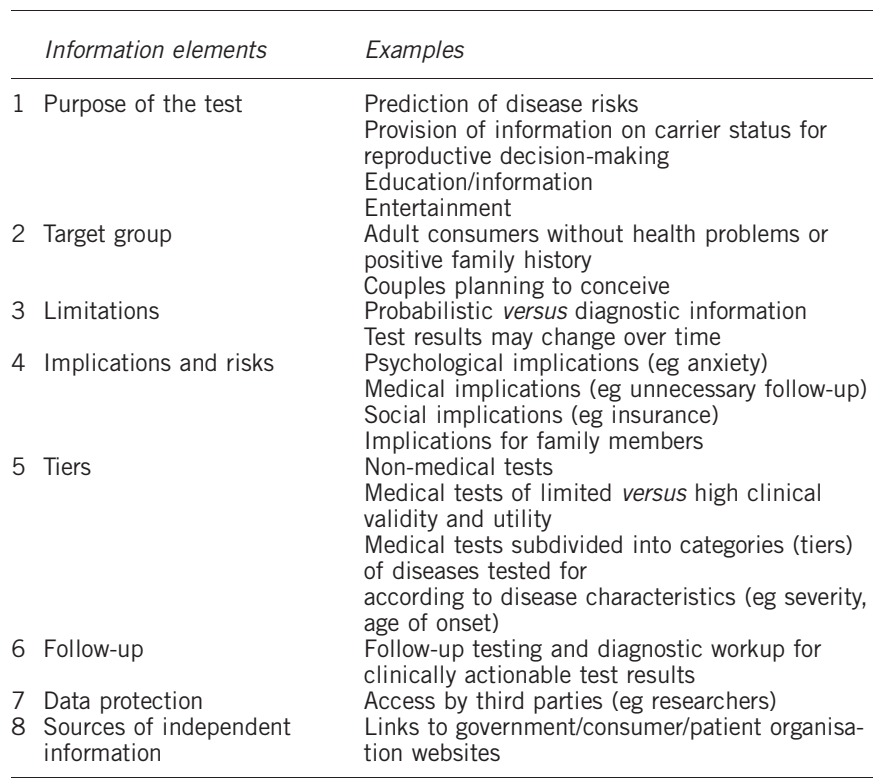


decodeme.com) or consumers who are interested in genetics and who wish to explore their genetic make-up out of curiosity. (23andme: http://www.23andme.com) $)^{34}$ Through an explicit definition of the target group, consumers with health problems and consumers who worry about genetic conditions that run in their families can be clearly informed that they may need to see a clinical geneticist instead of purchasing a PGT.

Further, in order to enhance autonomous choice, consumers should be given the opportunity to choose tiers so that they can opt out of categories of diseases (eg diseases for which there are no treatment options or psychiatric diseases). The company 23andme for example does this by offering test results for hereditary breast cancer, Alzheimer's disease and Parkinson's disease separately and asking separate informed consent for each of these three diseases. (23andme: http://www.23andme.com) Thus, the first layer of the information provision process contains information that is of direct relevance to the decision whether or not to proceed with PGT and to give informed consent, such as the purpose and the target group of the PGT, tiers from which to choose as well as key messages about the follow-up and general limitations, risks and implications of PGT test results.

Second and further layers may contain specific and detailed information about the tiers of the PGT and the individual diseases and traits tested for within these tiers, and about the clinical, personal and social significance and implications of test results within these tiers. Additional layers may further contain detailed information on the testing procedure, the laboratory analysis, the technology and algorithms used, the research findings on which risk calculations are based and references to the scientific literature. They may also contain additional information to improve understanding of the testing service, such as general facts about genetics, categories of complex diseases, disease prevention, genetic testing and its psychological and social implications, for those who seek explanation, explication or elaboration. Finally, they may contain legally relevant additional information such as the location of the laboratory, advertising and funding arrangements, details about policies for data security and for what will happen to the database if the company goes bankrupt. As the information offered in additional layers will be less crucial, the moral obligation to provide such information will be less stringent than the moral obligation to provide first-layer information. It follows that there may be reasons to leave the precise contents of additional layers to providers' discretion.

The proposed outline of a layered model is meant as a starting point for discussion. Geneticists, physicians, ethicists, policy-makers and commercial providers may need to work together to examine informational needs and preferences among consumers and to determine what information is material to informed consent in PGT. The aim of layered consent will now be clear: in order to avoid overwhelming consumers with information, only information that is essential to informed consent should be offered to all consumers in the first layer, whereas further layers of information provision are made available for those who desire more knowledge in order to consent. In other words: in the first layer, consumers are made aware of only the tips of all relevant icebergs, and they can find complete images of all icebergs in further layers if they wish.

\section{Staged consent}

The idea of staged consent underlines that informed consent is a process and takes time. People need time for consideration in order to come to understanding decisions. ${ }^{35}$ In clinical genetics, time is an essential feature of informed consent: counsellors allow for time to pass between counselling and decision-making. ${ }^{7}$ In the context of biobanks, stepwise informed consent is used to promote comprehension. ${ }^{36}$ In PGT, a staged set-up of the information provision process is already presupposed by a tiered model for informed consent: as different risks, limitations, follow-up and implications are associated with the different tiers, these tiers require separate discussions before tier-specific consent can be given. The tiered, layered and staged dimensions of the proposed model are thus not fully separable and will intertwine.

The process of purchasing a PGT can be subdivided into three informational and decisional phases, each to be concluded with informed consent: first, informed consent to the PGT as a whole or to certain tiers prior to buying the test; second, informed consent per tier prior to receiving the test results; and third, informed consent prior to receiving subsequent updates of test results. The general contention of the idea of staged consent is that the passing of time between these informational phases may allow for learning and improve understanding.

First, the process of pre-test information provision preparing for informed consent can be extended over time. The bulk of information need not be presented all at once, but can be subdivided into manageable portions, starting from general information about benefits and limitations of PGT, followed by introductory information about the tiers and concluded-upon demand-by specific information about the diseases tested for within selected tiers and associated risks and implications. This information may be repeated and built or elaborated upon and thus constitute a learning process. Models for a staged, integrated system of patient education and informed consent have been envisioned also in the clinical setting. ${ }^{37}$

PGT providers are already fulfilling part of the educational ideal of staging: they present a riches of information on their websites, (Counsyl: https://www.counsyl.com; Map My Gene: http://www.mapmygene.com.) including educational materials (http://www.23andme. com; deCODEme: http://www.decodeme.com) which can be accessed freely and repeatedly by anyone who has access to the Internet. Consumers can compare providers' websites, search for information, be taught the basics of genetics through tutorials, and in this manner compile and tailor their own pre-purchase information provision process. It remains important, however, that information material to the actual informed consent is expressly presented to all consumers (layered consent), and that informed consent is clearly requested.

Second, informed consent itself may be staged as test results may change over time. Many PGT companies offer their customers regular updates of their test results as new genetic variants come to be included in companies' risk profiles and original test results are reinterpreted, or as new associations are found between variants and diseases, thus expanding companies' testing offers. (23andme: http://www.23andme.com) Customers are given the opportunity to decide for each update whether or not they wish to view their new test results. (23andme: http://www.23andme.com) With these updates companies can rehearse or provide further information on diseases tested for, before asking customers to give renewed informed consent.

The proposed staged model emphasises that informed consent in PGT, in accordance with the science which underlies it, may undergo changes over time. Informed consent should be thought of as a process rather than a once-and-for-all-time transaction. For this reason, consumers should be able to withdraw from companies' databases and subscription lists. 
In conclusion, a tiered, layered and staged model for informed consent would structure the testing offer into tiers from which consumers may choose understandingly, would prioritise information such that consumers have sufficient knowledge of PGT generally and its associated risks, limitations and implications, and would allow for time to learn and time to consider. The structure of the Internet commonly facilitates multi-layered and staged design; like many other websites, PGT company websites are already layering information (eg 'click here to learn more'). (23andme: http://www.23andme. com $)^{16}$ With the help of information and communication technologies, processes of information provision and informed consent in PGT could be improved. It is not impossible that enabling and encouraging consumers to make more well-considered decisions regarding PGT will result in a smaller proportion of them consenting to and purchasing PGT. As such, adequate procedures for informed consent may run counter to companies' business interests. It is not yet known whether conflicts of interests will arise in practice. Regardless, however, the moral obligation remains the same: to mitigate the harms of testing and of misinterpretation and to protect the value of autonomous choice.

\section{CONCLUSION}

Providers of PGT can be argued to have general moral obligationsalso in a commercial context-to offer information about their services and to ensure that this information can be communicated effectively. In order to protect consumers against harm and to enable them to make autonomous choices, informed consent as an ethical requirement is indispensable. PGT commonly involves a lot of complex genetic information and thus poses difficulties for pre-test information provision and informed consent. This paper proposes a combined tiered-layered-staged model for informed consent that may serve as a response to widespread worries about misinterpretation and misunderstanding of PGT by consumers. The model is intended for the commercial and online context in which PGT is currently offered and in which face-to-face discussions with professional counsellors are lacking. The proposed model focuses on the consumer perspective, identifying the moments in which consumers make decisions about PGT and the information they likely need in order to make informed decisions about whether or not to consent to (certain tiers of) a PGT. The proposed contents of the model are no more than preliminary. In order to establish adequate tiered consent, academia, providers and consumers may collaborate on the definition of categories of diseases along the lines of clinical validity, purposes of testing, level of potential harm, and personal, clinical and social implications. Also, further research is needed to establish the exact contents of the first layer of the information provision process prior to informed consent, which is meant to contain only material, indispensable information on PGT. Ideally, an interactive approach would allow providers to check whether consumers have understood the key messages of PGT. A tiered-layered-staged model for informed consent can be applied to PGT in both direct-to-consumer and professionally mediated contexts, and help overcome the challenges regarding pre-test information provision and informed consent encountered in commercial PGT, now and in the future.

\section{CONFLICT OF INTEREST}

The authors declare no conflict of interest.

\section{ACKNOWLEDGEMENTS}

This text is a result of a research project of the Centre for Society and the Life Sciences in The Netherlands, funded by the Netherlands Genomics Initiative.
We thank all 13 participants to the expert meeting on informed consent held in May 2011, in Utrecht. We are indebted to one of the reviewers for the iceberg image in the paragraph on layered consent.

1 Council of Europe: Additional Protocol to the Convention on Human Rights and Biomedicine, concerning Genetic Testing for Health Purposes. Strasbourg, Council of Europe 2008.

2 European Society of Human Genetics (ESHG): Statement of the ESHG on direct-toconsumer genetic testing for health-related purposes. Eur J Hum Genet 2010; 18: 1271-1273.

3 Faden RR, Beauchamp TL: A History and Theory of Informed Consent. New York/ Oxford: Oxford University Press, 1986.

4 Manson NC, O'Neill O: Rethinking Informed Consent in Bioethics. Cambridge: Cambridge University Press, 2007.

5 Pranati: Informed consent: are we doing enough? Perspect Clin Res 2010; 1: 124-127.

6 Council of Europe: Convention for the Protection of Human Rights and Dignity of the Human Being with regard to the Application of Biology and Medicine. Oviedo, Council of Europe, 1997.

7 Kääriäinen $\mathrm{H}$, Hietala $M$, Kristoffersson $U$ et al: Recommendations for Genetic Counselling related to Genetic Testing. European Commission, EuroGentest 2008.

8 McBride CM, Bowen D, Brody LC et al: Future health applications of genomics: priorities for communication, behavioral, and social sciences research. Am J Prev Med 2010; 38: 556-565.

9 McGuire AL, Diaz CM, Wang T, Hilsenbeck SG: Social networkers' attitudes toward direct-to-consumer personal genome testing. Am J Bioeth 2009; 9: 3-10.

10 Bloss CS, Schork NJ, Topol EJ: Effect of direct-to-consumer genomewide profiling to assess disease risk. N Engl J Med 2011; 364: 524-534.

11 Howard HC, Borry P: Personal genome testing: do you know what you are buying? Am J Bioeth 2009; 9: 11-13.

12 Berg C, Fryer-Edwards K: The ethical challenges of direct-to-consumer genetic testing. J Bus Ethics 2007; 77: 17-31.

13 Gurwitz D, Bregman-Eschet Y: Personal genomics services: whose genomes? Eur J Hum Genet 2009; 17: 883-889.

14 Nuffield Council on Bioethics: Medical Profiling and Online Medicine: The Ethics of "Personalised Healthcare" in a Consumer Age. London, Nuffield Council on Bioethics 2010.

15 Human Genetics Commission (HGC): A common framework of principles for direct-toconsumer genetic testing services. London, HGC, 2010.

16 Lachance CR, Erby LAH, Ford BM, Allen VC, Kaphingst KA: Informational content, literacy demands, and usability of websites offering health-related genetic tests directly to consumers. Genet Med 2010; 12: 304-312.

17 Singleton A, Erby LH, Foisie KV, Kaphingst KA: Informed choice in direct-to-consume genetic testing (DTCGT) websites: a content analysis of benefits, risks, and limitations. J Genet Couns 2011; 21: 433-439.

18 Bunnik EM, Janssens ACJW, Schermer M: Informed consent in direct-to-consumer personal genome testing: the outline of a model between specific and generic consent. In press.

19 Elias S, Annas GJ: Generic consent for genetic screening. N Engl J Med 1994; 330 1611-1613.

20 O'Neill O: Some limits of informed consent. J Med Ethics 2003; 29: 4-7.

21 Beauchamp TL, Childress JF: Principles of Biomedical Ethics (fifth edition). New York, NY: Oxford University Press, 1994.

22 Eiseman E, Bloom G, Brower J, Clancy N, Olmsted SS: Case Studies of Existing Human Tissue Repositories: "Best Practices" for a Biospecimen Resource for the Genomic and Proteomic Era. Santa Monica, CA: RAND Corporation, 2003.

23 Entwistle VA, Carter SM, Trevena L et al: Communicating about screening. BMJ 2008; 337: 789-791.

24 National Bioethics Advisory Commission (NBAC): Research Involving Human Biological Materials: Ethical Issues and Policy Guidance. Springfield, VA, Department of Commerce 1999.

25 Ross LF: Mandatory versus voluntary consent for newborn screening? Kennedy Inst Ethics J 2010; 20: 299-328.

26 Bredenoord AL, Onland-Moret NC, Van Delden JJM: Feedback of individual genetic results to research participants: in favor of a qualified disclosure policy. Hum Mutat 2011; 32: 861-867.

27 Berg JS, Khoury MJ, Evans JP: Deploying whole genome sequencing in clinical practice and public health: meeting the challenge one bin at a time. Genet Med 2011; 13: 499-504.

28 Rizk C: James Evans' plan to make genetic testing data manageable for the clinic. Genomeweb 2011.

29 Bunnik EM, Schermer MHN, Janssens ACJW: The role of disease characteristics in the ethical debate on personal genome testing. BMC Med Genomics 2012 5. 4

30 US Department of Health and Human Services (HHS): Research-Based Web Design \& Usability Guidelines. Washington, DC, HHS 2006.

31 Kaphingst KA, McBride CM, Wade C, Alford SH, Brody LC, Baxevanis AD: Consumers' use of web-based information and their decisions about multiplex genetic susceptibility testing. J Med Internet Res 2012; 12: e41. 
32 Melzer D, Hogarth S, Liddell K, Ling T, Sanderson S, Zimmern RL: Genetic tests for common diseases: new insights, old concerns. BMJ 2008; 336: 590-593.

33 Thompson PA: Counterpoint: genetic risk feedback for common disease: time to test the waters. Cancer Epidemiol Biomarkers Prev 2007; 16: 1727-1729.

34 Su Y, Howard HC, Borry P: Users' motivations to purchase direct-to-consumer genomewide testing: an exploratory study of personal stories. J Community Genet 2011; 2: $135-146$.

35 National Commission for the Protection of Human Subjects of Biomedical and Behavioral Research: The Belmont Report: Ethical Principles and Guidelines for the Protection of Human Subjects of Research. Washington, DC: Department of Health, Education, and Welfare, 1979.
36 Mascalzoni D, Hicks A, Pramstaller P, Wjst M: Informed consent in the genomics era. PLoS Med 2008; 5: e192.

37 Tebbetts JB, Tebbetts TB: An approach that integrates patient education and informed consent in breast augmentation. Plast Reconstr Surg 2002; 110: 971-978.

(c) This work is licensed under the Creative ComSOMERTHHISHESERVIED mons Attribution-NonCommercial-No Derivative Works 3.0 Unported License. To view a copy of this license, visit http://creativecommons.org/licenses/by-nc-nd/3.0/ 